\title{
The Role of Custom-made Subperiosteal Implants for Rehabilitation of Atrophic Jaws - A Case Report
}

\author{
David Faustino Ângelo ${ }^{1,2}$, José Ricardo Vieira Ferreira² \\ ${ }^{1}$ Instituto Português da Face - Rua Tomás Ribeiro, Lisboa, Portugal, ${ }^{2}$ Universidade de Lisboa, Faculdade de Medicina - Avenida Professor Egas Moniz, \\ Lisboa, Portugal
}

\section{Abstract}

Rehabilitation of atrophic jaws with conventional endosseous implant-supported overdentures and immediate loading protocols still presents a challenge nowadays. Custom-made implants with immediate loading overdenture are emerging as a solution for atrophic jaws rehabilitation. The authors describe the case of a 44-year-old male with a history of congenital dental agenesis. A previous oral rehabilitation with an all-on-6 type, implant-fixed mandibular overdenture, had failed due to peri-implantitis. The patient was successfully treated with bimaxillary custommade subperiosteal implants with an innovative design, combining subperiosteal and endosseous support. The authors consider custom-made subperiosteal implants, in selected patients, present several advantages over classic bone-grafting plus endosseous implant-placement techniques such as (1) possibility of a single-stage procedure with immediate loading in atrophic jaws; (2) possible primary option to approach atrophic jaws as a simpler and less time-consuming technique; and (3) a valid rescue option for failed endosseous implants. More long-term studies with large samples of patients will be necessary to confirm previous assumptions.

Keywords: Atrophic jaws, bone atrophy, custom-made implants, oral rehabilitation, selective laser melting

\section{INTRODUCTION}

Rehabilitation of atrophic jaws with conventional endosseous implant-supported overdentures and immediate loading protocols still presents a clinical challenge nowadays. Many techniques have been described in the literature to overcome this problem. Reconstructive procedures, such as autologous bone grafting or guided bone regeneration, ${ }^{[1]}$ are often used. However, autogenous bone grafting requires a second surgical site, ${ }^{[2]}$ implying additional morbidity, ${ }^{[3]}$ and immediate loading is not always recommended. ${ }^{[4]}$ Guided bone regeneration, particularly vertical, is frequently limited in gain and also associated with possible complications in total atrophic jaws. ${ }^{[5]}$ Both techniques require several months for graft maturation. ${ }^{[6]}$ Alternative techniques for the rehabilitation of atrophic jaws, such as tilted implants ${ }^{[7]}$ and zygomatic implants, ${ }^{[8]}$ appear to provide stable long-term results. Atrophic jaws are associated with anatomical changes, carrying an increased risk of injury to noble structures, thus increasing the needs of specific surgical skills during surgery. Zygomatic implants procedures can be performed under local or general anesthesia, depending on the surgeon's experience and the patient condition. A favorable zygoma bone is essential to support the implant. ${ }^{[8]}$ On the severely

\begin{tabular}{|l|l|}
\hline \multicolumn{2}{|c|}{ Access this article online } \\
\hline Quick Response Code: & Website: \\
& www.amsjournal.com \\
\cline { 2 - 3 } & \\
&
\end{tabular}

atrophic jaw, the use of short implants remains controversial. ${ }^{[9]}$ Other techniques such as sinus lift, ${ }^{[10]}$ inferior alveolar nerve lateralization, ${ }^{[11]}$ or osteogenic distraction ${ }^{[12]}$ present diverse results in the literature. Custom-made subperiosteal implants are emerging nowadays as a solution for the rehabilitation of atrophic jaws, suitable for both maxillary atrophy and mandibular bone deficiencies. ${ }^{[13-15]}$ Several protocols have been proposed for subperiosteal implant techniques. Herein, the authors present their experience with an innovative design of custom-made subperiosteal implants that includes areas for endosseous support.

\section{Case Report}

A 44-year-old male with severe maxillary atrophy and previous mandibular all-on-6 rehabilitation, with current

Address for correspondence: Prof. David Faustino Ângelo Instituto Português da Face, № 71, $5^{\text {th }}$ Floor. 1050-227 Lisbon. Portugal. E-mail: david.angelo@ipface.pt
Received: 06-06-2020

Accepted: $15-09-2020$
Revised: 04-09-2020

Published: $23-12-2020$
This is an open access journal, and articles are distributed under the terms of the Creative Commons Attribution-NonCommercial-ShareAlike 4.0 License, which allows others to remix, tweak, and build upon the work non-commercially, as long as appropriate credit is given and the new creations are licensed under the identical terms.

For reprints contact: WKHLRPMedknow_reprints@wolterskluwer.com 
peri-implantitis, was referred to our department due to complaints of mobility both on the inferior implant-supported prosthesis and the superior prosthetic bridge, causing difficulty in chewing and speech. The patient reported a history of congenital dental agenesis, with complete mandibular rehabilitation with six implants $\left(\right.$ BioHorizon $^{\circledR}$ $3.5 \mathrm{~mm} \times 12 \mathrm{~mm}$ ) and a superior bilateral canine to central incisor prosthetic bridge, accomplished 24 years ago. The patient denied smoking habits or relevant systemic diseases, and his prosthetic goal was an immediate loading solution. Clinical examination and orthopantomography indicated a combined horizontal and vertical osseous deficiency and active peri-implantitis in all six implants previously placed in the inferior arch [Figure 1], confirmed through cone-beam computed tomography (CBCT) [Figure 2a and b]. Maxillary vertical and alveolar ridge deficiency was also noted [Figure $2 \mathrm{c}$ and d]. Bone quality was classified as Type III, with a thin layer of cortical bone surrounding dense of dense trabecular bone.
Bimaxillary custom-made implants were proposed, using the following protocol [Table 1]. An innovative design was used in the mandible including areas of endosseous support for adequate osseointegration.

\section{Stage 1 \\ 3-month preoperative: inferior implant removal and curettage}

Inferior implant removal with thorough bone curettage. Oral amoxicillin 875 + clavulanic acid $125 \mathrm{mg}$ was performed every $8 \mathrm{~h}$ for 3 months. A removable mucosa-supported prosthesis was applied exclusively for social needs during the preoperative period.

\section{Stage 2}

2-month preoperative: cone-beam computed tomographybased planning and custom-made implants design

Reverse planning was carried with the resulting DICOM data. Custom-made implants were designed by Bone Easy ${ }^{\circledR}$ with

\section{Table 1: Protocol for subperiosteal customized implant planning and treatment}

\begin{tabular}{l} 
Timeline \\
\hline $\begin{array}{l}\text { Stage } 1 \text { ( } 3 \text { months preoperative) - Preoperative inferior } \\
\text { implant removal and curettage }\end{array}$ \\
Stage 2 ( 2 months preoperative) - Preoperative CBCT-based \\
planning and custom-made implants design
\end{tabular}

planning and custom-made implants design

Stage 3 ( 1 month preoperative) - Custom-made subperiosteal implants design manufacturing

\section{Stage description}

Inferior implant removal with thorough bone curettage. Oral amoxicillin $875+$ clavulanic acid $125 \mathrm{mg}$ was performed every 8 hours for three months. A removable mucosa-supported prosthesis was applied exclusively for social needs

Reverse planning was carried with the resulting DICOM data. Custom-made implants were designed by Bone Easy ${ }^{\circledR}$ with inputs from the surgeon. Alveolar reduction was required to accommodate the bar, prosthetic components, and the prosthesis. A 3D-printed guide was designed for bone height reduction and endosseous fitting areas. Custom-made implants were designed with partial endosseous support to connect plates and suitable osseointegration (Figure 3). Implants were designed with a $0.7 \mathrm{~mm}$ thickness to adapt to the maxillary and mandibular buttresses through fixation with $2 \mathrm{~mm} \times 6 \mathrm{~mm}$ SLA treated osteosynthesis screws. Bone grafting was planned to be performed simultaneously with the placement of the mandibular implant, mostly in endosseous areas.

The implant was manufactured by selective laser melting using Truprint 1000 SLM machine, using Sintmill ${ }^{\circledR}$ to place the implants on an indexation framework for posterior mechanization. After printing the base plate, the implants were fixed by supports and submitted to heat treatment - 1hour heating to $800^{\circ} \mathrm{C}$ stabilized for 30 minutes and cooling for 4 hours. The frame and the implant were separated from the base and placed on a milling machine using SUM 3D software to make M2 threads and re-mechanization of the implant and abutment connection. The plates were polished on the surface that contacts soft tissue. The surface that contacts bone was left rough. The grafting zone was left unpolished. The alveolar reduction guide and the implant insertion guide were both manufactured using a 3D printer on medicalgrade plastic. All devices were sterilized with Ethylene Oxide before surgery

Surgery was performed under general anesthesia

On the maxilla, a crestal incision was performed from tuberosity to tuberosity, with one relieving incision in the midline. Buccal and palatal flaps were raised, exposing the anterior nasal spine, the pyriform apertures, the canine fossae, the zygomatic buttresses, and the posterolateral maxillae. Alveolar reduction was performed using a piezoelectric handpiece. The implant was tested and fixed with osteosynthesis screws On the mandible, a crestal incision was performed around the arch to the contralateral side. Care should be taken not to injure the neurovascular bundle. External oblique ridges, both mental foramina, mandibular symphysis, and genial tubercles, were identified and exposed to serve as anatomical landmarks. A large bur was used to design the endosseous support aided by a guide. The implant was tested, fixed with osteosynthesis screws, and bone grafting was carried in the endosseous zone (Figure 4) Abutments were placed, and flaps were closed with $4 / 0$ vicryl ${ }^{\circledR}$

Prosthetic impressions were taken immediately after closure, and a provisional prosthesis was successfully adapted $12 \mathrm{~h}$ later, prior to patient discharge 
inputs from the surgeon. Alveolar reduction was required to accommodate the bar, prosthetic components, and the prosthesis. A three-dimensional (3D)-printed guide was designed for bone height reduction and endosseous fitting areas. Custom-made implants were designed with partial endosseous support to connect plates [Figure 3]. Implants were designed with a $0.7 \mathrm{~mm}$ thickness to adapt to the maxillary and mandibular buttresses through fixation with $2 \mathrm{~mm} \times 6$ $\mathrm{mm}$ sandblasted, large grit, acid-etched implant surface (SLA) treated osteosynthesis screws. Bone grafting was planned to be performed simultaneously with the placement of the mandibular implant, mostly in endosseous areas.

\section{Stage 3}

\section{1-month preoperative: Custom-made subperiosteal implants design manufacturing}

The implant was manufactured by selective laser melting (SLM) using Truprint 1000 SLM machine, using Sintmill ${ }^{\circledR}$ to place the implants on an indexation framework for posterior mechanization. After printing the base plate, the implants were fixed by supports and submitted to heat treatment $-1 \mathrm{~h}$ heating to $800^{\circ} \mathrm{C}$ stabilized for $30 \mathrm{~min}$ and cooling for $4 \mathrm{~h}$. The frame and the implant were separated from the base and placed on a milling machine using SUM 3D software to make M2 threads and re-mechanization of the implant and abutment connection. The plates were polished on the surface that contacts soft tissue. The surface that contacts bone was left rough. The grafting zone was left unpolished. The alveolar reduction guide and the implant insertion guide were both manufactured using a 3D printer on medical-grade plastic. All devices were sterilized with ethylene oxide before surgery.

\section{Stage 4}

\section{Surgical procedure}

Surgery was performed under general anesthesia.

On the maxilla, a crestal incision was performed from tuberosity to tuberosity, with one relieving incision in the midline. Buccal and palatal flaps were raised, exposing the anterior nasal spine, the pyriform apertures, the canine fossae, the zygomatic buttresses, and the posterolateral maxillae. The alveolar reduction was performed using a piezoelectric handpiece. The implant was tested and fixed with osteosynthesis screws.

On the mandible, a crestal incision was performed around the arch to the contralateral side. Care should be taken not to injure the neurovascular bundle. External oblique ridges, both mental foramina, mandibular symphysis, and genial tubercles, were identified and exposed to serve as anatomical landmarks. A large bur was used to design the endosseous support aided by a guide. The implant was tested, fixed with osteosynthesis screws, and bone grafting was carried in the endosseous zone [Figure 4].

Abutments were placed, and flaps were closed with $4 / 0$ vicryl ${ }^{\circledR}$.

Prosthetic impressions were taken immediately after closure, and a provisional prosthesis was successfully adapted $12 \mathrm{~h}$ later, before patient discharge.

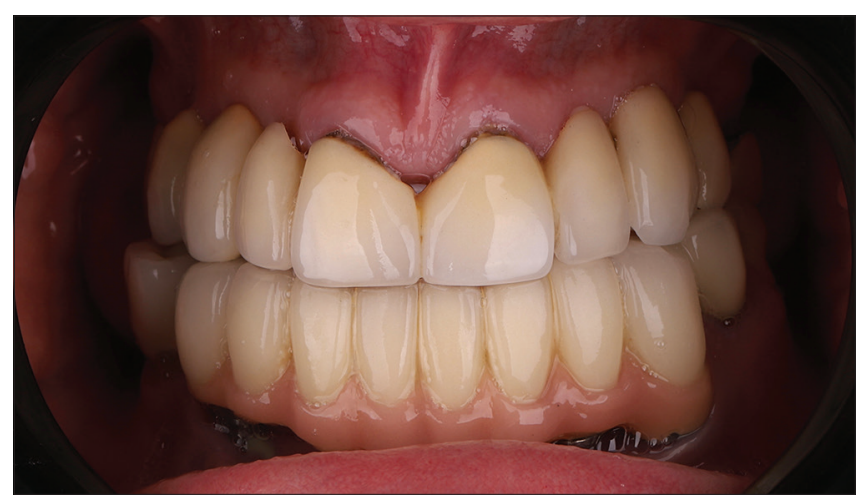

Figure 1: Preoperative dental photograph, highlighting inferior peri-implantitis
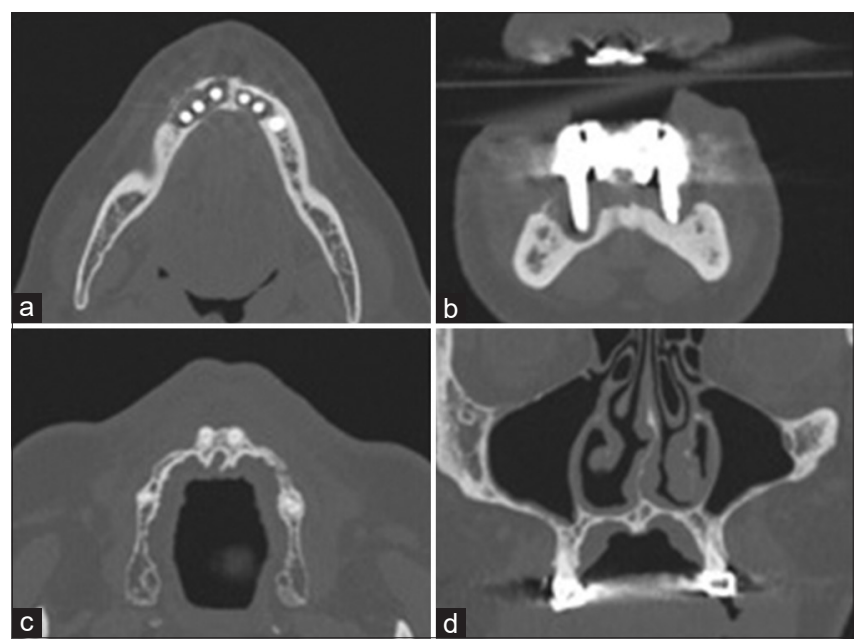

Figure 2: Cone beam computed tomography. (a) Axial plane, presenting six previously placed mandibular implants with active peri-implantitis. (b) Coronal plane, presenting previous mandibular implants with peri-implantitis. (c) Axial plane, presenting severe maxillary atrophy. (d) Coronal plane, presenting severe maxillary atrophy

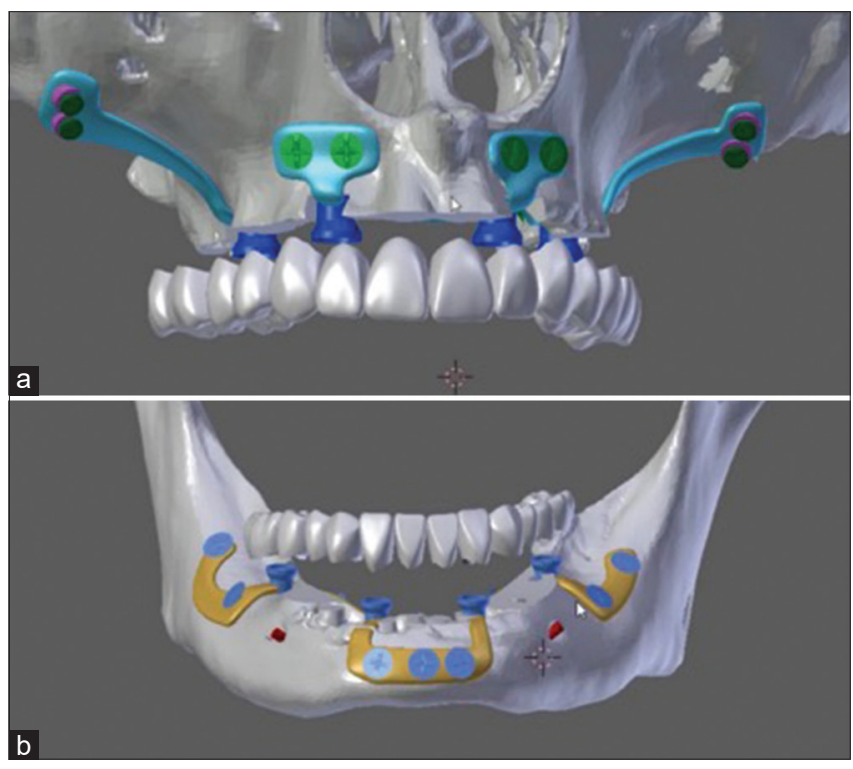

Figure 3: Reverse 3-D planning of the custom-made implant for maxilla (a) and mandible (b) 


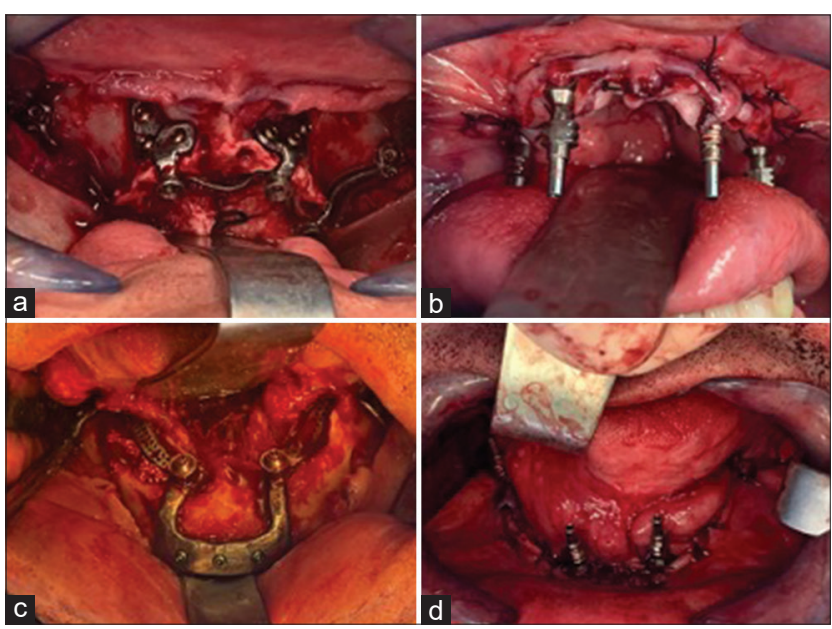

Figure 4: Surgical procedure. (a) Maxillary adaptation of the custom-made subperiosteal implant. (b) Prosthetic abutments adapted to the maxillary implant. (c) Mandibular adaptation of the custom-made subperiosteal implant with grafting zone in the endosseous area. (d) Prosthetic abutments adapted to the mandibular implant

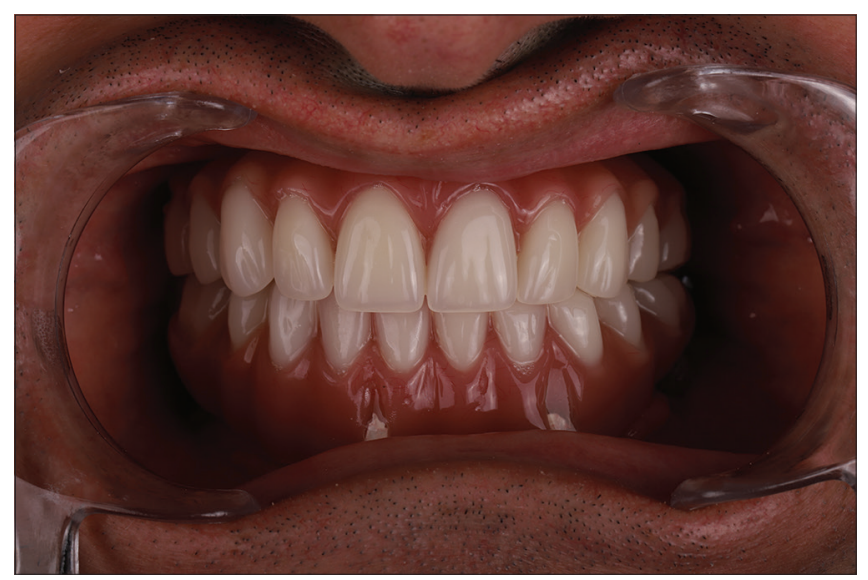

Figure 5: Postoperative dental photograph, 1-year follow-up time

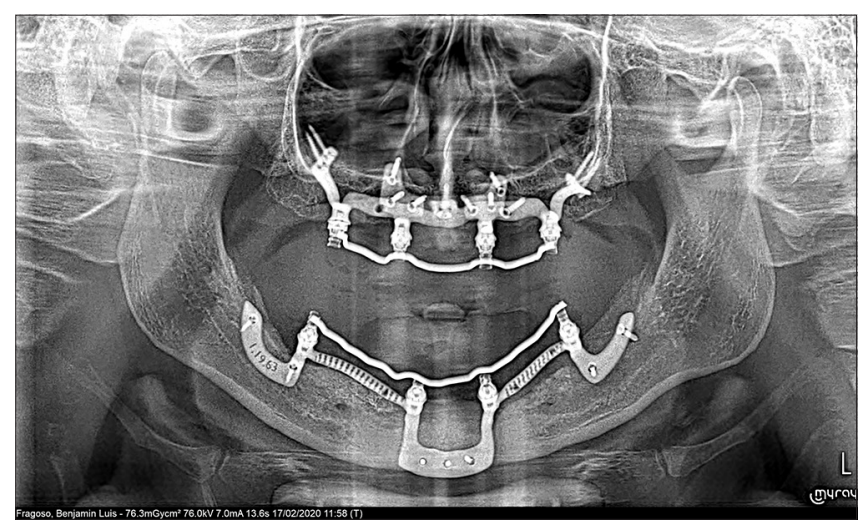

Figure 6: Postoperative orthopantomograph - 1-year follow-up

Connections were screw-retained, milled on the framework with $2.8 \mathrm{~mm}$ length. Tissue-level connections were used to receive a multiunit abutment with a $20^{\circ}$ cone to fix the prosthesis. The abutments used are compatible with most of the systems available in the market. Connections were planned to emerge on the usual position of canines and molars in each quadrant. Prostheses were made concerning bilateral occlusion balance, and a finite element study was performed to analyze the performance of the implant.

We report excellent implant stability, adequate masticatory function, no implant exposure, and absence of pain, over 1 year and 3 months of follow-up time [Figure 5]. A 1-year follow-up orthopantomograph is presented in Figure 6.

\section{Discussion}

Severe atrophic jaw dental rehabilitation remains a challenge for oral and maxillofacial surgeons. Lately, several techniques with good long-term results have been reported. However, with an increasing prevalence of peri-implantitis ${ }^{[16]}$ associated with endosseous implants, subperiosteal implants have presented as an alternative solution for full rehabilitation with immediate loading prosthesis. In the reported case, the patient presented with active mandibular peri-implantitis and thus was not suitable for treatment with endosseous implants, ${ }^{[17,18]}$ due to (1) high risk of new infection and (2) patient demand for immediate loading.

Subperiosteal implants were first described in 1943. ${ }^{[19]}$ However, they were soon associated with abnormal complication rates such as implant exposure, implant mobility, and implant loss. Recently, a digital revolution has been taking place in dentistry, related to new digital techniques for acquisition, improved processing software, and modern fabrication techniques, allowing for the beginning of a new era in fixed prosthodontics, including the customization of implant therapy. ${ }^{[15,20-22]}$

Cerea and Dolcini reported a series of seventy patients treated with custom-made direct metal laser sintering (DMLS) titanium subperiosteal implants that showed a survival rate of $95.8 \%$ and low complication rates over a 2-year follow-up period. They concluded that custom-made DMLS subperiosteal implants could present a valid alternative treatment procedure for prosthetic restoration of severely atrophic jaws, where the placement of endosseous implants is not possible. ${ }^{[13]}$

The presented solution is innovative, as it is custom-made to the patient's anatomy and designed to include endosseous support. It was fabricated in rigid Ti6Al4V through SLM technology and fixed with $2 \mathrm{~mm} \times 6 \mathrm{~mm}$ SLA-treated osteosynthesis screws. The authors believe that custom-made subperiosteal implants can be both an excellent rescue option and a valid first option to approach atrophic jaws, as a simpler and less time-consuming technique. Their main advantage resides in offering an alternative to more invasive surgical techniques such as iliac crest bone grafts and other bone augmentation procedures, as well as in allowing for immediate prosthetic loading. ${ }^{[13]}$ Their main problems could relate to (1) material fracture due to fatigue, (2) peri-implantitis, ${ }^{[14]}$ (3) implant exposure, (4) implant mobility, (5) lack of osteointegration, ${ }^{[22]}$ and (6) length of the connection pillars used, which might 
predispose to fractures of both the implant and of the prosthetic restoration. ${ }^{[13]}$ So far, the authors did not notice any of those possible complications in this case. However, more long-term studies with larger samples of patients will be necessary to establish this technique further.

\section{Acknowledgments}

We are grateful to Bone Easy ${ }^{\circledR}$ engineers, who contributed to the surgical planning, and to Rita Nascimento, DDS, responsible for prosthetic rehabilitation.

\section{Declaration of patient consent}

The authors certify that they have obtained all appropriate patient consent forms. In the form the patient(s) has/have given his/her/their consent for his/her/their images and other clinical information to be reported in the journal. The patients understand that their names and initials will not be published and due efforts will be made to conceal their identity, but anonymity cannot be guaranteed.

\section{Financial support and sponsorship}

Nil.

\section{Conflicts of interest}

There are no conflicts of interest.

\section{REFERENCES}

1. Buser D, Dula K, Hess D, Hirt HP, Belser UC. Localized ridge augmentation with autografts and barrier membranes. Periodontol 2000 1999;19:151-63.

2. Albrektsson T. Repair of bone grafts. A vital microscopic and histological investigation in the rabbit. Scand J Plast Reconstr Surg 1980;14:1-2.

3. Nkenke E, Radespiel-Tröger M, Wiltfang J, Schultze-Mosgau S, Winkler G, Neukam FW. Morbidity of harvesting of retromolar bone grafts: A prospective study. Clin Oral Implants Res 2002;13:514-21.

4. Kim A, Kar K, Nowzari H, Cha HS, Ahn KM. Immediate free iliac bone graft after nonsegmental mandibular resection and delayed implant placement: A case series. Implant Dent 2013;22:438-43.

5. Cucchi A, Vignudelli E, Napolitano A, Marchetti C, Corinaldesi G. Evaluation of complication rates and vertical bone gain after guided bone regeneration with non-resorbable membranes versus titanium meshes and resorbable membranes. A randomized clinical trial. Clin Implant Dent Relat Res 2017;19:821-32.
6. Sethi A, Kaus T, Cawood JI, Plaha H, Boscoe M, Sochor P. Onlay bone grafts from iliac crest: A retrospective analysis. Int J Oral Maxillofac Surg 2020;49:264-71.

7. DE Vico G, Bonino M, Spinelli D, Schiavetti R, Sannino G, Pozzi A, et al. Rationale for tilted implants: FEA considerations and clinical reports. Oral Implantol (Rome) 2011;4:23-33.

8. Aparicio C, Manresa C, Francisco K, Ouazzani W, Claros P, Potau JM, et al. The long-term use of zygomatic implants: A 10-year clinical and radiographic report. Clin Implant Dent Relat Res 2014;16:447-59.

9. Al-Ansari A. Short implants supporting single crowns in atrophic jaws. Evid Based Dent 2014:15:85-6.

10. Silva L de F, de Lima VN, Faverani LP, de Mendonça MR, Okamoto R, Pellizzer EP. Maxillary sinus lift surgery - With or without graft material? A systematic review. Int J Oral Maxillofac Surg 2016;45:1570-6.

11. Vetromilla BM, Moura LB, Sonego CL, Torriani MA, Chagas OL Jr., Complications associated with inferior alveolar nerve repositioning for dental implant placement: A systematic review. Int J Oral Maxillofac Surg 2014;43:1360-6

12. Günbay T, Koyuncu BÖ, Akay MC, Sipahi A, Tekin U. Results and complications of alveolar distraction osteogenesis to enhance vertical bone height. Oral Surgery Oral Med Oral Pathol Oral Radiol Endodontology 2008;105:e7-13.

13. Cerea M, Dolcini GA. Custom-made direct metal laser sintering titanium subperiosteal implants: A retrospective clinical study on 70 patients. BioMed Res Int 2018;2018:11.

14. Nguyen TM, Caruhel JB, Khonsari. A subperiosteal maxillary implant causing severe osteolysis. J Stomatol Oral Maxillofac Surg 2018; 119:523-525.

15. Surovas A. A digital workflow for modeling of custom dental implants. 3D Print Med 2019;5:9.

16. Tarnow DP. Increasing prevalence of peri-implantitis: How will we manage? J Dent Res 2016;95:7-8.

17. Molinero-Mourelle P, Baca-Gonzalez L, Gao B, Saez-Alcaide LM, Helm A, Lopez-Quiles J. Surgical complications in zygomatic implants A systematic review. Med Oral Patol Oral Cir Bucal 2016;21:e751-e757.

18. Robertson K, Shahbazian T, MacLeod S. Treatment of peri-implantitis and the failing implant. Dent Clin North Am 2015;59:329-43.

19. Dahl G. Om mojligheten for inplantation i kaken av metallskelett som bas eller retention for fasta eller avtagbara proteser. Odontol Tidskr 1943.

20. Joda T., Zarone F., Ferrari M. The complete digital workflow in fixed prosthodontics: A systematic review. BMC Oral Health 2017;17:124.

21. Buser D, Sennerby L, De Bruyn H. Modern implant dentistry based on osseointegration: 50 years of progress, current trends and open questions. Periodontology 2000;73:2017, 7-21.

22. Claffey N, Bashara H, O’Reilly P, Polyzois I. Evaluation of new bone formation and osseointegration around subperiosteal titanium implants with histometry and nanoindentation. Int J Oral Maxillofac Implants 2015;30:1004-10. 